ALPHA Nº 27 Diciembre 2008 (63-76)

ISSN 0716-4254

http://alpha.ulagos.cl

\title{
LENGUAJE, ABSTRACCIÓN Y SÍMBOLO EN LA NARRATIVA DE FELISBERTO HERNÁNDEZ
}

Language, abstraction and symbol in Felisberto Hernández's narrative works

Joaquín Lameiro Tenreiro*

Resumen

A partir de la formulación de un modelo teórico y su posterior aplicación a textos concretos, se lleva a cabo una investigación sobre la naturaleza del lenguaje literario entendido como verbalización de un objeto abstracto por medio de una función símbolo y sobre las particularidades de esta naturaleza a través de una revisión del concepto de "metonimia" en la narrativa del escritor uruguayo Felisberto Hernández (1902-1964).

Palabras clave: Felisberto Hernández, lenguaje, abstracción, símbolo, metonimia.

Abstract

By the means of the development of a theoretical model and its further application to concrete texts, this paper makes its point on the nature of literary language which is understood as the verbalization of an abstract object by means of a "function-symbol" and on the particularities of this nature in Uruguayan writer Felisberto Hernández's narrative works throughout a revision of the term "metonymy".

Key words: Felisberto Hernández, language, abstraction, symbol, metonymy.

Las relaciones entre lenguaje, abstracción y símbolo que definen la obra narrativa de Felisberto Hernández se presentan como un ejemplo del intento de superación de la crisis del lenguaje por parte de la Vanguardia histórica. Esta crisis, inmanente al lenguaje desde sus mismas bases, se traduce en el concepto de inefabilidad: el lenguaje, tradicionalmente entendido en su función comunicativa-denotativa, no es capaz de expresar lo tradicionalmente calificado como "inefable", que tiene que ver con un conocimiento intuitivo y no sistemático del yo y del mundo, porque esta expresión necesita la creación de un objeto abstracto. Parece, por tanto, evidente que lo inefable quedaría fuera del ámbito de lo lingüístico, por cuanto ese objeto abstracto no es susceptible de "ser comunicado", sino que debe ser producido en cada acto expresivo. Sin embargo, si partimos de una revisión de los términos "lenguaje” y "abstracción” atendiendo no a criterios 


\section{Joaquín Lameiro Tenreiro}

mentalistas o instrumentales, sino materialistas y absolutos, podremos observar cómo la expresión de lo inefable a través del lenguaje se hace no sólo posible, sino esencial tanto en el ejercicio literario de Hernández como en el conjunto de las expresiones artísticas que renovaron el acto lingüístico humano durante la primera mitad del siglo XX. ${ }^{1}$

En primer lugar, se suele asociar el concepto de "abstracción" con una naturaleza mentalista, o incluso idealista, cuando lo cierto es que se trata de un fenómeno material, basado en la disociación entre una expresión y un contenido y, por tanto, explicable como una función estructuradora del conocimiento. En segundo lugar, se ha sostenido - y se sostiene- - una naturaleza instrumental del fenómeno lingüístico, entendiendo el lenguaje como una herramienta intrínsecamente humana para comunicarse con otros miembros de una misma comunidad a través de una denotación referencial. ${ }^{2}$ Se puede llegar a pensar que, siendo el lenguaje un instrumento comunicativo-denotativo y la abstracción un proceso “desligado", existe una relación de incompatibilidad entre ambos. Esto es así debido a una concepción del lenguaje excesivamente limitada a cuya ampliación este artículo pretende contribuir.

Si entendemos el lenguaje en términos generativo-transformacionales, ${ }^{3}$ es decir, como la capacidad de un individuo para crear una infinidad de enunciados nuevos a partir de un conjunto finito de objetos lingüísticos y de reglas recursivas de generación y transformación, hemos de observar que esta definición es válida sólo para una "clase" de lenguaje, esto es, aquél que se refiere a la tradicionalmente denominada "facultad lingüística humana” y cuya materialización es comunicativa y denotativa. No obstante, si llevamos nuestra concepción del lenguaje un poco más allá, podemos llegar a alcanzar la perspectiva de un sistema válido en sí mismo y despojado de las

\footnotetext{
${ }^{1}$ Con respecto a las relaciones entre inefabilidad y lenguaje, Adorno advierte que, "el lenguaje significativo quisiera decir el absoluto a través de la mediación, y se le escurre en cada intención singular, abandona cada una tras de sí por limitada. La música lo encuentra de forma inmediata, pero en el mismo instante se oscurece, como un exceso de luz que deslumbra los ojos, de tal modo que lo completamente visible ya no puede ser visto” (2000:28). Aunque no se tratarán en este artículo, las relaciones entre música y literatura en la obra de Hernández han sido ampliamente explotadas (Cfr. especialmente, Giraldi, 1998) y contribuyen a explicar, por lo menos en cierta medida, el proceso de verbalización de lo abstracto del que se tratará aquí.

${ }^{2}$ Así ha sido estudiado el lenguaje desde Saussure por estructuralistas y funcionalistas y, en cierta medida, también por la Gramática Generativo-Transformacional, si bien ésta introduce un concepto que resulta interesante en mi análisis: el de la "creatividad" del hablante, que presenta evidentes paralelismos con el concepto de "productividad” que utilizaré más adelante, siguiendo a Kristeva.

${ }^{3}$ Creo que debemos entenderlo en estos términos si queremos superar la barrera descriptivista del estructuralismo y el funcionalismo.
} 
condiciones de comunicatividad y denotación, que constituye una práctica productora de textos eximidos de cualquier otro referente que no sea el propio lenguaje. ${ }^{4}$

Este lenguaje, que denominaremos "productivo", se materializa en aquel otro lenguaje comunicativo y denotativo, a través de un proceso de verbalización que provoca un desfase insalvable en tanto que el lenguaje denotativo fracasa en su tarea de asignar un referente (una denotación) al texto generado por el lenguaje productivo. En términos de cognición humana y lingüística, se puede hablar de un fallo de interpretación del texto por parte del mecanismo lingüístico, que Chomsky (1999) denomina el "componente computacional". Es decir, que un texto no comunicativo/no denotativo verbalizado en una lengua natural exige un acceso directo al sistema cognitivo, sin que su estructura superficial pueda ser interpretada eficazmente por el componente computacional. Este texto productivo verbalizado que hace fracasar la interpretación lingüística (morfológica, sintáctica y semántica) y que reclama una interpretación cognoscitiva tiene su principal representante en el "texto poético" $\mathrm{y}$, hasta bien entrado el siglo $\mathrm{XX}$, venía siendo estudiado como una variación retórica de las lenguas naturales. Es a partir de los estudios de semiótica cuando se empieza a comprender su naturaleza profunda como práctica autónoma respecto a las estructuras lingüísticas verbalizables.

El texto poético se genera, pues, a través de una entidad que permite aunar el objeto abstracto creado por el "lenguaje productivo" a una estructura normalizada verbalmente a través de un desplazamiento de la formulación lingüística productiva (irreductible por sí misma a la expresión) hacia la formulación lingüística verbalizada (expresable). Esta entidad puente es, en realidad, una función al modo de la anáfora de Kristeva (1978: Vol. I, Cap. 5.I, 118-129) que, a través de recursos metafóricos y metonímicos (y en este sentido hablo de desplazamiento) realiza un trabajo de transformación sobre el texto, de forma que un texto de naturaleza productiva (material e inexpresable, en este sentido) es "trasladado" mediante una función

\footnotetext{
${ }^{4}$ En este sentido, este artículo se plantea como una relectura de Kristeva (1978) intentando actualizar una teoría que, pese a su valor intrínseco, se ha ido soterrando bajo una ideología marxista excesivamente marcada que la ha sumido en un cierto desfase histórico con el paso de los años.

${ }^{5}$ Entiendo por texto poético, de acuerdo con Kristeva (1978), un texto que no cumple las normas por las que se rige el uso lingüístico común o canónico. En mi opinión, esta exclusión de la normatividad es lo que provoca que el texto poético sea ininterpretable en una clave puramente lingüística, es decir, propia de un sistema de reglas finitas.
} 


\section{Joaquín Lameiro Tenreiro}

transformadora hacia lo material expresable en virtud de una verbalización. ${ }^{6}$ A esta función transformadora la denominaré símbolo, por cuanto aúna dos realidades irreductibles en una misma expresión. Hemos de notar que se entiende el símbolo, entonces, como una función (y no como una unidad) que relaciona dos etapas del proceso mental: la de una estructura inicial no verbalizable y la de una estructura final verbalizada. ${ }^{7}$ Esta función aparece siempre que y sólo cuando la estructura inicial no es verbalizable, lo que no sucede en los procesos comunicativo-denotativos — cuya verbalización se rige por mecanismos morfo-sintáctico-semánticos- sino en procesos (en textos) productivos, esto es, en textos poéticos. ${ }^{8}$

En el caso de Hernández, la función símbolo adquiere una idiosincrasia muy particular al formularse estrictamente en términos metonímicos: ${ }^{9}$ frente al mecanismo sustitutivo inducido por la metáfora, la metonimia se establece en la prosa de Hernández como una estrategia desplazante y es por ello que representa una función simbólica muy elaborada. Diríamos, casi, "purista", pues, en definitiva, la metáfora se formula siempre a partir de una referencialidad (una metarreferencialidad, si se prefiere) en la que el elemento lingüístico A' viene a sustituir a un elemento lingüístico A que no es, en realidad, sino un referente lingüístico. Hernández encuentra serios problemas a la hora de aceptar esta metarreferencialidad como vehículo para verbalizar

\footnotetext{
${ }^{6}$ Conviene justificar mi concepción de lo material como aquello opuesto a lo ideal. En este sentido, rechazo la explicación idealista del lenguaje como un sistema de modelos inmateriales que se materializan en la subjetividad del hablante. Esta visión, de raíces platónicas, desvía el sentido que quiero dar a mi concepción del lenguaje productivo, como una práctica inmanente al individuo y que se genera en unas condiciones históricas materiales.

${ }^{7}$ Evito aquí los términos de estructura profunda y estructura superficial, en primer lugar, porque no se refieren a lo mismo, ya que ambos se mantienen en el ámbito del lenguaje comunicativo (por lo que toda estructura profunda convergente es verbalizable) y, en segundo término, porque el propio Chomsky (1999) ha eliminado en su teoría más reciente la estructura profunda. En todo caso, la estructura superficial sí se podría asimilar a mi estructura final verbalizada. Sin embargo, nótese que toda función se ejecuta dentro de una estructura. En este sentido, sí cabría hablar de una estructura de la producción de textos. El tema de la estructura productiva o creativa ha encontrado especial relevancia en la Filosofía de la Música. John Cage, en "Conferencia sobre nada", dice que la nada (que en el contexto particular de tal Conferencia, tiene mucho que ver con una idealización de la estructura) es "como un vaso de leche. Necesitamos el vaso y necesitamos la leche. O incluso es como un vaso vacío en el que en cualquier momento podemos verter cualquier cosa” (2002:110).

${ }^{8}$ Entiendo, por tanto, el texto como un proceso productivo, y no como un producto, por cuanto el texto se presenta como un ser orgánico y en continua interacción con la Historia.

${ }^{9}$ Adviértase que el uso de la metonimia en Hernández es sobradamente conocido. Lo que aquí se pretende es trascender el nivel descriptivista para hallar una justificación interpretativa de ese uso.
} 
su texto productivo, ${ }^{10}$ y así lo expresa, por medio de un mecanismo concretivo, en su conocido texto "El taxi”

El precio de la metáfora ha aumentado demasiado para mi bolsillo. Aprovecho las circunstancias de que el "varita" hace señal de que los vehículos se detengan; pero mis ideas siguen: ellas han comprendido que ya han estado demasiado tiempo adentro y que si no quiero que me encierren, deben trabajar para afuera. Entonces, he abierto de pronto la portezuela del taxi y me he perdido entre la multitud. ${ }^{11}$

El narrador evita el taxi/metáfora en la medida en que - como vehículo de alquiler - supone sólo una sustitución $\mathrm{y}$, en este sentido, impide que las "ideas", el objeto abstracto, entren en contacto con "la multitud". Se establece, así, la metáfora como una función simbólica unívoca, que tan sólo "encierra" una referencia metalingüística, frente a un contacto plural ("multitudinario") con la materia abstracta a la que sólo se accede mediante la aproximación directa, es decir, la metonimia.

$\mathrm{Si}$ "El taxi” se presenta como un texto casi programático, no descuidará Hernández la praxis de tal programa. Así, El corazón verde, uno de los cuentos incluidos en el volumen Nadie encendía las lámparas, traduce a la práctica literaria no ya el rechazo a la metáfora como ejercicio falsamente productivo, sino la preponderancia de la metonimia como verdadero desplazamiento del lenguaje denotativo hacia la inefabilidad del lenguaje productivo. El título, El corazón verde, ofrece la clave para interpretar el relato como una "poética práctica" de la metonimia: la estructura narrativa se construye a partir de un objeto tangible - un alfiler de corbata - que aglutina diversos recuerdos en los que él ha estado presente. Esto es, el alfiler se desplaza materialmente por la historia del personaje y, metonímicamente, por la historia del narrador. Nos encontramos, además, con que personaje y narrador son coincidentes y, por tanto, la materialidad de la metonimia se hace todavía más patente

\footnotetext{
10 De hecho, la metáfora no introduce un texto productivo, pues no promueve un desplazamiento entre los niveles productivo y denotativo, sino una sustitución de un elemento denotativo por otro “de segundo grado”. Ya Bajtin (1989) hace notar este agotamiento de la tradicional retórica de tropos en lo que concierne a la palabra novelesca, pues considera inapropiada una retórica orientada "hacia los géneros unilinguales y monoestilísticos, hacia los géneros poéticos, en el sentido restringido de la palabra” (84).

${ }^{11}$ Felisberto Hernández. Obras Completas. Edición al cuidado de María Luisa Puga, 3 vols. México D.F.: Siglo XXI, 2000 (Vol. I), 2005 (Vol. II), 2005 (Vol. III), I, 102. Citaremos por esta edición.
} 


\section{Joaquín Lameiro Tenreiro}

Primero ese alfiler había sido una pequeña piedra verde que el mar había desgastado dándole forma de corazón; después la habían puesto en un prendedor y el corazón había quedado emplomado entre un cuadrilátero del tamaño de un diente de caballo. Al principio, mientras yo lo daba vuelta entre mis dedos, pensaba en cosas que no tenían que ver con él; pero de pronto él me empezó a traer a mi madre, después a un tranvía a caballos, una tapa de botellón, un tranvía eléctrico, mi abuela, una señora francesa que se ponía un gorro de papel y siempre estaba llena de plumitas sueltas, su hija, que se llamaba Ivonne y le daba un hipo tan fuerte como un grito, un muerto que había sido vendedor de gallinas, un barrio sospechoso de una ciudad de la Argentina y donde en un invierno yo dormía en el suelo y me tapaba con diarios, otro barrio aristocrático de otra ciudad donde yo dormía como un príncipe y me tapaba con muchas frazadas, y, por último, un ñandú y un mozo de café (II, 149:150).

Se observa, con especial claridad en este pasaje, cómo funciona el proceso metonímico de asociación entre texto productivo y texto denotativo.

En primer lugar, se establece una referencialidad denotativa extremadamente materialista, de forma que el planteamiento de la anécdota comienza con una descripción pormenorizada del elemento sobre el que recae la función símbolo, el alfiler de corbata, en su aspecto puramente material. Una vez establecido el nexo, el fragmento de materia que pondrá a narrador y lector en contacto con lo inefable, se procede a llenar este nexo de cargas simbólicas (experienciales y subjetivas y, por ello, a priori irreducibles a una formulación lingüística comunicativa) por medio de la aproximación física en la anécdota y asociativa en la estructura del relato, del alfiler hacia las experiencias de inefabilidad. Básicamente, el cuento, en tanto estructura simbólica por aproximación metonímica, queda reducido al párrafo citado. El resto de las aproximadamente ocho páginas que conforman el texto se reduce a un "comentario"12 de la función símbolo, que acaba de verbalizar en términos plenamente interpretables el texto productivo; pero, lo cierto es que todo el andamiaje estructural, temático y, sobre todo, sémico del discurso queda completo ya en la formulación embrionaria.

Si bien, en el caso de este cuento la función símbolo se efectúa a través de una metonimia objetual $\mathrm{y}$, en este sentido, el alfiler representa la codificación de una isotopía del texto por medio de un acercamiento del

12 Es obligado hacer notar aquí la importancia del concepto de “comentario” en Hernández, entendido como exégesis de su propio texto, como autointerpretación ya de su obra, ya de su experiencia vital en cuanto material literario. A este respecto, $C f r$., por ejemplo, La envenenada (I, 69-79). 
elemento abstracto isotópico (esto es, el tema del relato) hacia un elemento semántico plenamente materializado en la superficie textual (un leitmotiv), lo cierto es que el narrador hernandiano suele resolverse por estructuras más complejas en cuanto a las relaciones metonímicas que en ellas se establecen, y así bosqueja su narración en un devenir de pensamiento asociativo que busca su coherencia en una justificación por proximidad de los distintos elementos que activan el relato. En El caballo perdido, el narrador, a través de los ojos del niño que fue, recuerda un incidente con la fotografía de un hombre a la que "se enfrenta" en casa de su maestra de piano

Había sacado rápidamente la mirada de los ojos de él [del hombre retratado] y la había colocado rígidamente en sus bigotes. Después del engorde negro que tenía encima de la boca salían para los costados en línea recta y seguían así un buen trecho. Entonces pensé en los dedos de mi abuela: eran gordos, rechonchos — una vez se pinchó y le saltó un chorro de sangre hasta el techo- - y aquellos bigotes parecían haber sido retorcidos por ella. (Ella se pasaba mucho rato retorciendo con sus dedos transpirados el hilo negro para que pasara la aguja; y como veía poco y para ver mejor echaba la cabeza hacia atrás y separaba demasiado el hilo y la aguja de los ojos, aquello no terminaba nunca). Aquel hombre también debió haberse pasado mucho rato retorciéndose el bigote; y mientras hacía eso y miraba fijo, quién sabe qué clase de ideas tendría (II, 15).

Observamos en esta cita cómo el motor de la narración es el pensamiento asociativo que, mediante relaciones metonímicas por proximidad, rompe las barreras entre cada núcleo semántico del texto, entrelazándolos, "enhebrándolos”, de modo que el hilo que va tejiendo el relato no es el del devenir de la anécdota, sino la propia relación entre una anécdota y otra en la memoria y en la imaginación narrativas, con el peligro explícitamente enunciado de que "aquello no acabara nunca”.

Pero la función simbólica por desplazamiento no se ofrece siempre a través de un desplazamiento efectivo que halle correspondencias exactas entre un elemento "productivo" y su correspondiente "denotativo", o bien traduzca la isotopía que hila los distintos momentos del relato. Por el contrario, la mayor parte de los textos de Hernández se elabora a partir del desplazamiento simbólico de una inefabilidad, generalmente un proceso emotivo-cognitivo, ${ }^{13}$ no hacia un objeto o leitmotiv, sino hacia una situación que constituye la

\footnotetext{
${ }^{13}$ En este sentido, Hernández se vale, sobre todo, del proceso de rememoración y del análisis sobre dicho proceso para elaborar los “objetos emotivo-cognoscitivos” a los que luego dará forma textual.
} 


\section{Joaquín Lameiro Tenreiro}

anécdota del relato. Desplazamiento que, asimismo, no se contenta con una simple ilación metonímica de las distintas anécdotas, actuando a un nivel más profundo, como una archi-metonimia que traduce la abstracción emotivocognitiva en un texto único y trabado. La labor del lector consiste, entonces, en traspasar la frontera de lo denotativo-anecdótico (y, con ella, la frontera del absurdo y de la banalidad actanciales que barniza la superficie narrativa) para abrirse camino hasta el elemento inefable, hasta la profundidad emotiva y epistemológica que reside, como velada por el desplazamiento verbal, tras la anécdota simbólica. Y, entonces, el símbolo se convierte en una forma de entrevisión, en una materialidad translúcida de lo que en realidad se intenta verbalizar, a través de los blancos en la anécdota. Esto es, toda una serie de ambigüedades premeditadas que el narrador suspende de su relato - y en las que la condición anecdótica queda suspendida - y por las que el lector encuentra una puerta de acceso a lo inefable: el conocido comienzo de Por los tiempos de Clemente Colling no se nos debe aparecer, ahora, tan inocente, tan deudor de una estética naïve - como hasta hace no tanto tiempo se venía sosteniendo- sino, muy al contrario, como una declaración ética, perfectamente honesta, del rechazo de los mecanismos sustitutivos en virtud de una operación desplazante que, provocando un desfase entre enunciación y producción, deja abierta, precisamente por medio de este desfase, una grieta en el texto que el lector utiliza para alcanzar, casi diríamos sin mediación, lo profundo del lenguaje productivo

No sé bien por qué quieren entrar en la historia de Colling, ciertos recuerdos. No parece que tuvieran mucho que ver con él. La relación que tuvo esa época de mi niñez y la familia por quien conocí a Colling, no son tan importantes en este asunto como para justificar su intervención. La lógica de la hilación (sic.) sería muy débil. Por algo que yo no comprendo, esos recuerdos acuden a este relato. Y como insisten, he preferido atenderlos (I, 138).

Es decir, el magma inefable que subyace a la superficie textual en forma de "recuerdo" está constantemente pujando para invadir la anécdota, y el narrador se ve en la obligación (ética antes que estética) de dejarlo salir. Pero, no se permite una intromisión abrumadora del recuerdo, sino que "prefiere atenderlo", erigiéndose en "negociador frente a sí mismo"14 y

\footnotetext{
${ }^{14}$ Este desdoblamiento del narrador en sí mismo y en el negociador de sí mismo supone el dilema que impide el progreso de la narración en El caballo perdido: el "socio" del "yo" y el "yo" entran en conflicto y desconfían el uno del otro, de forma que la negociación se rompe y el relato queda suspendido. Es con la reconciliación entre "socio” y "yo" que el "yo" encuentra
} 
dosificando, modelando este desplazamiento de lo inefable hacia su verbalización. Por ello, el narrador prosigue

Además tendré que escribir muchas cosas sobre las cuales sé poco; y hasta me parece que la impenetrabilidad es una cualidad intrínseca de ellas; tal vez cuando creemos saberlas, dejamos de saber que las ignoramos; porque la existencia de ellas es, acaso, fatalmente oscura: y ésa debe ser una de sus cualidades.

Pero no creo que solamente deba escribir lo que sé, sino también lo otro (I, 138).

El narrador se propone, pues, desplazar su constructo narrativo hacia lo inefable, hacia lo "impenetrable", para que el relato sirva como velo revelador, como pintura de lo invisible y, en definitiva, como símbolo por acercamiento del imposible epistemológico.

Por todo lo que se ha expuesto, resulta evidente que en Hernández la estética del relato es vicaria de una ética de lo otro, de lo inefable, que se materializa en una función metonímica que vincula la estructura narrativa al objeto abstracto que se quiere verbalizar, a través del concepto programático del "escribir lo otro". ${ }^{15}$ Esta escritura de la otredad se manifiesta, ya, desde los primeros textos de Hernández, ${ }^{16}$ pero, es a partir de su etapa de madurez cuando sus narradores consiguen desligarse casi por completo de un protagonismo egocéntrico y autobiográfico que lastraba, en buena medida, el salto a la otredad para convertirse en narradores-testigos no ya de sí mismos, como ocurría en las nouvelles: Por los tiempos de Clemente Colling, El caballo perdido y Tierras de la memoria, ${ }^{17}$ sino en testigo de las actividades y actitudes de los otros. El punto crítico de esta alienación del narrador —que, desde luego, nunca llega a cumplirse enteramente- se cristaliza en los relatos que conforman Nadie encendía las lámparas, la única colección que Hernández concibió como tal. Encontramos aquí que, a través de una

su lugar en "el mundo" (real y narrativo) y —en una posición privilegiada que le permite salir de sí mismo sin alienarse completamente- puede culminar el proceso narrativo.

${ }^{15}$ Nótese que habla Hernández de "escribir lo otro", y no “escribir de/sobre lo otro”, y cómo esta transitividad insiste en el carácter plástico, material y directo del acceso metonímico a lo abstracto.

${ }^{16}$ Ya en el texto primerizo La piedra filosofal (I, 26-31), bajo una estructura narrativa muy sencilla, se perfila el acercamiento a la otredad por medio del desplazamiento: el narrador cede su voz a una piedra y será ésta la que esté capacitada para enunciar una serie de teorías que el narrador, mero transcriptor del discurso, desconoce.

${ }^{17}$ Aunque en esta última se aprecia claramente la voluntad escrutiñadora del "yo” narrativo, no sólo frente a sí mismo, sino con respecto a los otros. Probablemente el narrador no ha olvidado el aprendizaje crítico de la segunda parte de El caballo perdido (Cfr. nota 13). 


\section{Joaquín Lameiro Tenreiro}

excentración compasiva, el narrador-testigo modela una serie de caracteres ajenos que perfilan, en cuanto personajes de una trama, un desplazamiento hacia lo profundo del relato que se concreta en una representación del "misterio" — esto es, de lo inefable; de lo otro- por medio de una actitud extravagante por la cual, en efecto, el narrador, más que sentir simpatía, se compadece. ${ }^{18}$ Si ya en las tres nouvelles, Hernández nos acercaba a lo inasible de su propio misterio - al objeto abstracto de su propia mismidad a través de un acerado sentimiento de autocompasión- es ahora cuando la compasión se vuelca sobre el otro, provocando así un giro radical desde la introspección hacia la observación, fenómeno que propicia un mayor trabajo de la anécdota como vehículo que concreta el proceso metonímico, al centrarse ahora la experiencia narrativa en un contar antes que en un analizar lo contado.

Resultará útil, pues, analizar brevemente esta concreción de lo abstracto por medio de la observación del otro en alguno de los cuentos de Nadie encendía las lámparas. Ya el cuento que abre y da título al volumen supone un ejemplo muy clarificador. El narrador-protagonista (protagonista en cuanto observador y, en todo caso, "emanador" del "misterio" que luego materializarán los otros personajes) se sitúa en el centro del relato: es un escritor y pianista invitado a una velada en una casa burguesa y, en este sentido, los personajes giran en torno a él, tanto por su faceta de narrador en el relato como de "escritor invitado" en el espacio actancial de la casa. El tema del cuento es, como señala Morillas, el surgimiento del deseo y la contraposición entre ese deseo posible y el aburrimiento actual

La proyección de su propia escisión [del narrador-protagonista] en actor distraído/sujeto deseante, orienta la búsqueda: la de lo latente y entrevisto, soñado e imaginado, escindiendo los objetos, cosificando a sus personajes, seleccionando y enfatizando lo aspectual (2000:40).

Pero, lo que aquí interesa es que esta "escisión”, misterio o abstracción que pugna por emerger a la superficie textual, ya no lo hará por la vía de un objeto - un leitmotiv - como veíamos en El corazón verde, que forma parte también de esta colección, ni como un hilo isotópico que aúna los distintos fragmentos de la narración — como en el ejemplo analizado de El caballo

${ }^{18}$ Considero muy importante el hecho de que los narradores de Hernández tienen grandes dificultades para empatizar o simpatizar con ellos mismos, con sus narraciones y con sus personajes, entrando por el contrario en una dialéctica de la compasión —o la autocompasiónque, en gran medida, es una de las dificultades a las que el lector se debe enfrentar, por cuanto debe entrar en esa dialéctica de la compasión que, por lo demás, resulta bastante incómoda dentro de una tradición literaria basada eminentemente en la relación empática entre autor, lector y obra. 
perdido — sino que, aun sin descuidar estas estrategias, la verbalización de lo abstracto a través de la situación anecdótica adquiere su asidero más sólido en un personaje - que lo es tanto en su faceta actancial como, especialmente, simbólica- sobre el que el narrador-protagonista proyecta el "misterio", el deseo. Se trata de una joven, sobrina de las dueñas de la casa

Ella sonrió y bajó los ojos. Entonces yo pude mirarle toda la boca, que era muy grande. El movimiento de los labios, estirándose hacia los costados, parecía que no terminaría más; pero mis ojos recorrían con gusto toda aquella distancia de rojo húmedo. Tal vez ella viera a través de los párpados; o pensara que en aquel silencio yo no estuviera haciendo nada bueno, porque bajó mucho la cabeza y escondió la cara. Ahora mostraba toda la masa del pelo; en un remolino de las ondas se le veía un poco de la piel, y yo recordé a una gallina que el viento le había revuelto las plumas y se le veía la carne. Yo sentía placer en imaginar que aquella cabeza era una gallina humana, grande y caliente; su calor sería muy delicado y el pelo era una manera muy fina de las plumas (II, 57-58).

Adviértase cómo el desplazamiento de la abstracción del deseo hacia su concreción verbal se realiza siempre de un modo dinámico, siendo en este sentido plenamente metonímico. En un primer momento, es el movimiento de la mujer el que materializa el deseo; pero, luego - y esto es lo más interesante - la asociación entre la cabeza de la joven y una gallina se establece a través de un "movimiento de pensamiento" y se recalca la actancialidad de la función símbolo por medio de la forma "yo recordé a una gallina”, de modo que el sujeto portador de lo abstracto ("yo") y la verbalización de lo abstracto hacia el plano superficial ("una gallina”) se conjugan en una acción verbal de movimiento, de desplazamiento mental: “recordé”. Y es por ello que hablamos, en el caso de Hernández, de la preponderancia del mecanismo metonímico sobre el metafórico en lo que se refiere a la producción de la función símbolo en sus textos. ${ }^{19}$

Para concluir, queda retomar esta estrategia sistemática de desplazamiento del texto verbal hacia el texto productivo mediante la metonimia y hacer recapitulación de ella. No únicamente para establecer una taxonomía

\footnotetext{
${ }^{19}$ Esta misma función por desplazamiento a través de personajes e imágenes puede observarse claramente en otros textos de la colección. Así, en El balcón, es de nuevo una joven la que concreta la "angustia” del narrador-testigo mediante su relación cuasi-amorosa con el balcón de verano de su cuarto; en Menos Julia, un amigo de infancia introduce al narrador-testigo en su “enfermedad de tocar”, materializada esta vez en un túnel. Vemos, así, que los desplazamientos metonímicos siguen la línea "narrador-personaje-objeto" con una recurrencia muy marcada.
} 
descriptiva de sus ocurrencias sino, en grado más importante, para justificar de forma definitiva la ética que impulsa el proceder estético de Hernández. Hemos comenzado por dilucidar el proceso de aproximación entre dos niveles a priori incompatibles en un texto (el inefable y el verbal) como una operación de "traducción" de lo inefable a términos verbales no plenamente interpretables a nivel gramatical, traducción a través de la que se abre un acceso directo a la plena interpretación del conjunto (del texto en su totalidad) que ya no necesita ceñirse a una interpretación gramatical, en virtud, paradójicamente, a esta verbalización. Hemos sostenido que esta operación es una función que denominamos "simbólica" y que halla su fundamento en una aplicación extendida y polivalente de los mecanismos de sustitución y de desplazamiento del lenguaje (metáfora y metonimia, respectivamente). Este primer paso ha servido para allanar el terreno sobre el que luego analizaríamos el proceso de verbalización en la narrativa de Felisberto Hernández.

En los textos del uruguayo, la función símbolo se ofrece en una casuística explícitamente metonímica, lo que viene a justificar su estética como un quehacer literario apoyado, especialmente, en el concepto de desplazamiento, tanto en el nivel de la consolidación de una coherencia textual más allá de los operadores lógicos tradicionales del discurso como en el más complejo de adecuación del texto verbalizado al texto productivo. Debemos, ahora, intentar justificar cuál es la intención última de Hernández a la hora de establecer y proclamar esta estética del desplazamiento. La respuesta se revela coherente si nos detenemos a reflexionar sobre lo que la crítica ha escrito con respecto a su tratamiento del objeto: Hernández $-\mathrm{y}$ en este aspecto la crítica es unánime - tiende en su narración a "liberar" el objeto del dominio antropocéntrico del sujeto. ${ }^{20}$ Por lo que concierne al lenguaje, y en este sentido al texto como constructo productivo verbalizado, su estrategia de desplazamiento metonímico sólo puede justificarse dentro de este propósito ético de liberación del objeto: el desplazamiento metonímico no sustituye un lenguaje por otro - como sí lo hace la metáfora, que se prefigura como una referencialidad metalingüística - sino que objetiva el lenguaje; lo libera del sujeto productor y lo hace productivo en sí mismo, como materialización más inmediata y más veraz de lo abstracto. Pero esto, en realidad, no es sino lo que el joven narrador de Por los tiempos de Clemente Colling ha aprendido de su maestro ciego $-\mathrm{y}$, por ello, él mismo objetivado, exento en gran medida de la realidad convencional, de ese

${ }^{20}$ Como muestra de esta afirmación, puede confrontarse el detallado análisis que a este respecto se elabora en Valcárcel (1996). 
lenguaje de la imagen que el vidente acepta sin reflexión- cuando, en una de las últimas ocasiones en las que ve a Colling, asiste a una objetivación del proceso poético en términos radicales

Así como el sentido de lo nuevo - cuando yo llegaba a un país que no conocía - de pronto se me presentaba en ciertos objetos — las formas de las cajas de cigarrillos y fósforos, el color de los tranvías (y no siempre el espíritu muy diferenciado de las gentes) - Colling me dio un sentido nuevo de la vida con muchas clases de objetos. Yo observaba sus hechos, sus sentimientos, el ritmo de sus instantes, como otros objetos, o con sorpresa de objetos. Una noche yo iba a la escalera y en una oscuridad densa él trabajaba en el cajón de su mesa. En cada uno de los cuatro rincones tenía una pila de figuritas de cajas de fósforos y en cada figurita había una fórmula de armonía hecha en puntos. Hacía con ellos combinaciones que nunca pude comprender. Él me decía que en un rincón estaba la música de cámara, en otro la de ópera, en otro la de instrumentistas y en otro música sinfónica. Esa noche había hecho tan extrañas combinaciones, que llegó a decirme: ¿Sabe una cosa? que tiene razón Stravinski, Prokofieff, Ud. y todos los locos como Ud. (I, 195196).

Y así es, en efecto, cómo Felisberto Hernández nos muestra el sentido de lo nuevo: trasladándolo a las cajas de fósforos lingüísticas que componen sus relatos; objetivándolo para que esa "sorpresa de objetos" nos haga entrar de lleno en la materialidad de lo abstracto. De la misma y exacta manera como el narrador descubre el misterio de las nuevas ciudades o Colling se convence de la "razón" de la vanguardia musical rusa, así, Hernández nos conduce a través de la fantasía de sus relatos, hecha ya no carne, sino objeto, materia que es verbo pero que, al mismo tiempo, se libera de su propia condición verbal, en pujanza con el autor, lector y con el lenguaje mismo.

Universidade da Coruña* Profesor Investigador FPU Facultad de Filología Departamento de Filología Española y Latina Campus da Zapateira, s/n, 15071, A Coruña (España)

jlameiro@udc.es 
Joaquín Lameiro Tenreiro

\section{BIBLIOGRAFÍA}

HERNÁNDEZ, Felisberto. Obras Completas. Edición al cuidado de María Luisa Puga, 3 vols. México D. F.: Siglo XXI Editores, 2000 (Vol. I), 2005 (Vol. II), 2005 (Vol. III).

ADORNO, Theodor W. "Música, lenguaje y su relación en la composición actual”, en Sobre la música. Trad. Marta Tafalla González y Gerard Vilar Roca. Barcelona: Paidós, 2000.

BAJTIN, Mijail. Teoría y estética de la novela. Trad. Helena S. Kriúkova y Vicente Cazcarra. Madrid: Taurus, 1989.

CAGE, John. "Conferencia sobre nada”, en Silencio. Trad. Marina Pedraza. Madrid: Árdora, 2002.

CHOMSKY, Noam. El Programa Minimalista. Trad. Juan Romero Morales. Madrid: Alianza, 1999.

GIRALDI DEI CAS, Nora. Felisberto Hernández, musique et littérature. París: Indigo \& Côté-femmes éditions, 1998.

KRISTEVA, Julia. Semiótica. Trad. José Martín Arancibia. Madrid: Fundamentos, 1978.

MORILLAS, Enriqueta. "Prólogo” a su edición de Felisberto Hernández. Nadie encendía las lámparas. Madrid: Cátedra, 2000.

VALCÁRCEL, Eva. "La representación de la fábula surreal: Nadie encendía las lámparas de Felisberto Hernández”, en De Baudelaire a Lorca. Acercamiento a la modernidad literaria. Kassel (Alemania): Reichemberg, 1996. 